\title{
Capacitação de agentes comunitários de saúde para identificação de casos de tuberculose em uma unidade saúde da família
}

\author{
Training of community health agents to identify tuberculosis cases in a family health unit
}

Formación de agentes de salud comunitarios para identificar casos de tuberculosis en una unidad de salud familiar

Gabriella Sousa de Oliveira ${ }^{1 *}$, Ana Catarina da Paz Holodniak ${ }^{1}$, Bianca Abdelnor Hanna Piqueira Diniz¹, Ruan Seguin Azevedo Quaresma¹, Márcia Gomes Alcântara1, Karen Lury Abe Emoto, Gabriela Ladeia da Silva ${ }^{1}$, Danilo Rocha de Aguiar ${ }^{1}$, Ravena Larissa dos Santos Jardim¹, Rodrigo Silva Dias ${ }^{1}$.

\section{RESUMO}

Objetivo: Capacitar os Agentes Comunitários de Saúde de uma ESF de Belém, para o reconhecimento de pacientes suspeitos de tuberculose. Métodos: Estudo quantitativo, descritivo, transversal e analítico, realizado em uma Estratégia de Saúde da Família (ESF) da cidade de Belém - PA. A aplicação do trabalho ocorreu em três momentos: aplicado o questionário como pré-teste, palestra sobre tuberculose ministrada pelas pesquisadoras e reaplicação do instrumento de coleta como pós-teste. As variáveis quantitativas foram avaliadas por meio do teste T-Student $(p<0,05)$. Resultados: Na aplicação do pré-teste, evidenciou que todos os participantes possuem domínio nos eixos temáticos no que tange à definição, modo de transmissão, quadro clínico e prevenção da tuberculose. Nos demais tópicos, identificou-se uma discrepância no conhecimento prévio entre os participantes e os eixos com maior dificuldade de conhecimento foram relacionados ao tratamento diretamente observado (TDO) em 55,5\% ( $n=5$ ) e etiologia da TB com $44,4 \%(n=4)$. Os resultados obtidos no pós-teste constatam uma equivalência nos conhecimentos adquiridos possuindo significância estatística ( $p$ valor $=0,027$, Média $\pm 1,77$; $D P \pm 1.98$ ). Conclusão: A maioria ACS da ESF estudada mesmo com relatos verbais de que possuiam muitas dúvidas em relação ao tema, os resultados desta intervenção são considerados satisfatórios.

Palavras-chave: Agentes comunitários de saúde, Conhecimento, Tuberculose.

\begin{abstract}
Objective: To train Community Health Agents in an ESF in Belém, for the recognition of patients suspected of tuberculosis. Methods: Quantitative, descriptive, transversal and analytical study, carried out in a Family Health Strategy (FHS) in the city of Belém - PA. The work was applied in three moments: the questionnaire was applied as PRE-TEST, a lecture on tuberculosis given by the researchers and reapplication of the collection instrument as POST-TEST. Quantitative variables were assessed using the T-Student test ( $p$ $<0.05)$. Results: In the application of the PRE-TEST, it was evidenced that all the participants have mastery in the thematic axes regarding the definition, mode of transmission, clinical picture and prevention of tuberculosis. In the other topics, a discrepancy in prior knowledge was identified between the participants and the axes with the greatest difficulty in knowledge were related to the directly observed treatment (DOT) in $55.5 \%(n=5)$ and TB etiology with $44.4 \%(n=4)$. The results obtained in the POST-TEST show an equivalence in the knowledge acquired, having statistical significance ( $p$ value $=0.027$, Mean $\pm 1.77 ; S D \pm$ 1.98). Conclusion: Most FHS ACS studied, even with verbal reports that they had many doubts regarding the theme, the results of this intervention are considered satisfactory.
\end{abstract}

Keywords: Community health works, Knowledge, Tuberculosis.

${ }^{1}$ Centro Universitário Metropolitano da Amazônia (UNIFAMAZ), Belém - PA.

*E-mail: gsousa.do@gmail.com 
RESUMEN

Objetivo: capacitar a agentes de salud comunitaria en un FSE en Belém, para el reconocimiento de pacientes sospechosos de tener tuberculosis. Métodos: Estudio cuantitativo, descriptivo, transversal y analítico, realizado en una Estrategia de Salud de la Familia (FHS) en la ciudad de Belém - PA. El trabajo se aplicó en tres momentos: el cuestionario se aplicó como PRE-TEST, una conferencia sobre tuberculosis impartida por los investigadores y la reaplicación del instrumento de recolección como POST-TEST. Las variables cuantitativas se evaluaron mediante la prueba T-Student $(p<0.05)$. Resultados: en la aplicación de PRE-TEST, se evidenció que todos los participantes tienen dominio en los ejes temáticos con respecto a la definición, el modo de transmisión, el cuadro clínico y la prevención de la tuberculosis. En los otros temas, se identificó una discrepancia en el conocimiento previo entre los participantes y los ejes con mayor dificultad en el conocimiento se relacionaron con el tratamiento observado directamente (DOT) en $55.5 \%$ (n $=5)$ y la etiología de la tuberculosis con $44.4 \%(n=4)$. Los resultados obtenidos en el POST-TEST muestran una equivalencia en el conocimiento adquirido, con significancia estadística (valor $p=0.027$, media $\pm 1.77 ; \mathrm{SD} \pm 1.98$ ). Conclusión: la mayoría de los FHS ACS estudiados, incluso con informes verbales de que tenían muchas dudas con respecto al tema, los resultados de esta intervención se consideran satisfactorios.

Palabras clave: Agentes comunitarios de salud, Conocimiento, Tuberculosis.

\section{INTRODUÇÃO}

A tuberculose é descrita como uma das patologias mais antigas que se tem notícia na história, no entanto, o termo tal como se descreve a doença só começou a ser utilizado no ano de 1839 . No contexto mundial, cerca de 10,4 milhões de pessoas adoeceram por tuberculose no ano de 2015, com 1,4 milhão de mortes pela doença (LEÃO RNQ, 2013).

A Organização Mundial de Saúde (OMS) estima que, aproximadamente, um terço da população mundial tenha infecção latente por bactérias pertencentes ao complexo Mycobacterium tuberculosis. Deste grupo, 8 a 10 milhões de casos de tuberculose surgem a cada ano, e dentre esses, cerca de $50 \%$ dos casos são caracterizadas como formas infectantes da doença (BRASIL, 2017).

As regiões mais endêmicas no mundo incluem as nações insulares do Pacífico (exceto Japão), a Ásia, o subcontinente indiano, a África subsaariana e a América Latina, a Rússia e as antigas Repúblicas Soviéticas. Por conta de tratamento obsoleto, inadequado ou não disponível, 2 a 3 milhões de pessoas por ano morrem devido à tuberculose (GOLDMAN L, et al., 2005; BRASIL, 2015).

O Brasil faz parte do grupo dos 30 países de alta carga priorizados pela Organização Mundial da Saúde (OMS) e estes países concentram $87 \%$ dos casos de tuberculose no mundo, sendo que o Brasil ocupa a $16^{a}$ posição em número absoluto de casos. No país, no período de 2005 a 2014 foram diagnosticados 73 mil casos novos de tuberculose, e no ano de 2015, foram identificados 69 mil casos novos de tuberculose, com 4,5 mil mortes em decorrência desta patologia, caracterizando a TB como a terceira causa de morte por doença infecciosa na população geral e a primeira causa de morte entre doenças infecciosas nas pessoas que convivem com HIV/Aids (BRASIL, 2017).

A região Norte aparece como destaque com 67.000 casos, e possui cerca de 6000 mil novos casos notificados a cada ano. Dentro desta região, destaca-se o Estado do Pará, e mais especificamente a cidade de Belém, apresentando cerca de 1.300 novos casos novos de tuberculose pulmonar a cada ano, o que representa $45 \%$ do número total de notificações no país (SAMPAIO SN, et al., 2013).

Uma das problemáticas relacionadas à TB é a não captação de todos os casos pelos sistemas de vigilância epidemiológica. O número total de casos que ocorre a cada ano é estimado globalmente, assim como para as regiões e países, gerando dúvidas quanto a seguridade das informações de carga da doença. As ausências de notificações podem ser atribuídas às pessoas que não procuram o serviço de saúde ou 
procuram atendimento, no entanto, não recebem o diagnóstico adequadamente (WORLD HEALTH ORGANIZATION, 2012). Para reduzir esse déficit de informação de diagnóstico, notificação e incidência, recomenda-se o fortalecimento do acesso, diagnóstico e vigilância.

É imprescindível que todos os portadores de baciloscopia positiva sejam tratados e acompanhados mensalmente de modo a avaliar a evolução da sua situação de saúde, bem como minimizar os riscos de transmissão. Durante o período de atividade da doença, um paciente pode infectar dez outros indivíduos, em média, o que permite a perpetuação da cadeia de transmissão da tuberculose na comunidade (OLIVEIRA GP, et al., 2010).

Para atender a esta demanda, o Ministério da Saúde criou o Programa Nacional de Controle da Tuberculose (PNCT) que está contido na Estratégia Saúde da Família - ESF, e que com o auxílio Programa de Agentes Comunitários de Saúde (PACS), trabalham para a expansão das ações de controle da TB no território (BERALDO AA, et al., 2012).

É de suma importância que as equipes de saúde da família estejam qualificadas para reconhecer os possíveis pacientes sintomático respiratórios, e que esta equipe conte também com um bom suporte laboratorial para atender a demanda da sua área de cobertura, principalmente aquelas lotadas em níveis periféricos da rede de atenção de saúde (ROGERIO WP, 2015).

No contexto da busca ativa dos casos, a figura do agente comunitário de saúde - ACS caracteriza-se como um elo entre a comunidade e os demais membros da equipe da ESF na implementação de ações de promoção e vigilância em saúde. Por residirem na localidade em que atuam, conhecem a situação local e possuem proximidade com as famílias de suas micro áreas, agindo de modo a construir pontes entre os serviços de saúde e a comunidade, identificando prontamente os problemas desta, e facilitando o trabalho de prevenção de doenças e a promoção da saúde (FAÇANHA MC, et al., 2009).

De acordo com o Ministério da Saúde, é papel do ACS divulgar para sua comunidade a tuberculose como importante problema de saúde pública atual e as ações de controle no combate à TB precisam estar inseridas no planejamento das equipes de saúde do território.

Dessa forma, cabe ao ACS, durante a interação comunidade e principalmente durante a visita domiciliar, orientar a população quanto ao modo transmissão da doença e sobre as medidas de que podem ser adotadas para prevenir a TB, bem como a identificação de pacientes sintomáticos respiratórios, fazendo o devido encaminhamento destes até a unidade de saúde, devendo ser uma estratégia priorizada pelos ACS (ROGERIO WP, 2015).

No município de Belém existem 678 ACS em exercício registrados no Cadastro Nacional de Estabelecimentos de Saúde, segundo dados de dezembro de 2017 Estes agentes atuam na realização de atividades de prevenção de doenças e na promoção de saúde. Para o exercício profissional é pré-requisito residir na área da comunidade na qual trabalha, ter o Ensino Fundamental completo e possuir o curso introdutório de formação inicial e continuada (ROCHA GSS, et al., 2015).

Das habilidades e conhecimentos exigidos à atuação do ACS, destaca-se a capacidade de articulação entre os saberes técnicos e populares. Desse modo, o conhecimento sobre a tuberculose e o modo como estes profissionais podem agir para promover o controle da doença, resulta na execução das tarefas dos ACS de forma satisfatória (BRASIL, 2018).

No intuito de orientar os agentes comunitários de saúde sobre a TB, o Ministério da Saúde, em parceria com a Secretaria de Políticas de Saúde, elaborou uma cartilha que contém orientações diversas e extremamente importantes na identificação e controle da doença, facilitando o trabalho dos ACS no seu território e esclarecendo dúvidas durante a visita domiciliar de forma objetiva (ROGERIO WP, 2015).

É valido ressaltar que foram evidenciados poucos estudos nacionais que tentam mensurar o conhecimento de profissionais de saúde em relação à TB ou mesmo que evidenciem a importância do ACS na busca do paciente sintomático respiratório, a falta do treinamento adequado para o reconhecimento dos casos configura-se como um entrave para a identificação dos casos suspeitos (SCATOLIN BE, 2014). 
Conforme os dados estatísticos apresentados pelo Ministério da Saúde, deve-se dar importância na capacitação do agente comunitário de saúde para o auxílio na identificação de pacientes sintomáticos respiratórios, visto que a tuberculose ainda é doença muito prevalente em nossa realidade. Vale ressaltar que o diagnóstico precoce e o correto seguimento do tratamento propiciam ao paciente a cura completa e a quebra da cadeia de transmissão, por sua vez, diminuindo as chances de recidiva e as possíveis complicações decorrentes da falta ou seguimento inadequado do tratamento ao paciente com tuberculose (SAMPAIO SN, et al., 2013).

Em dias atuais, a tuberculose - TB ainda continua preocupando as autoridades sanitárias, apesar dos conhecimentos científicos abrangentes disponíveis sobre a doença e o desenvolvimento de estratégias de controle na saúde pública.

O Sistema de Informação de Agravos de Notificação - SINAN é o principal instrumento nacional para coleta e análise dos dados de TB. Entretanto, há limitações que dificultam o conhecimento real da doença, como a subnotificação, a baixa completude dos dados e a precariedade das informações relacionadas ao encerramento dos casos de TB, principalmente dentro das unidades saúde da família, dificultando a execução de atividades que busquem o controle da patologia.

Diante desta realidade, o presente trabalho se propôs em realizar a capacitação de agentes comunitários de saúde para identificação de casos de tuberculose em uma Unidade de Saúde da família, situado na periferia do município de Belém-PA, visando o auxílio na identificação de novos casos, estabelecendo o diagnóstico precoce e tratamento adequado, consequentemente, quebrando a cadeia de transmissão e reduzindo os óbitos causados por esta patologia. Além de fornecer melhoria na alimentação dos dados epidemiológicos sobre tuberculose na referida Unidade de Saúde.

\section{MÉTODOS}

Trata-se de um estudo quantitativo, descritivo, transversal e analítico, realizado em uma Estratégia de Saúde da Família (ESF) da cidade de Belém - PA, no período do mês de maio de 2018. A população alvo foi composta pelos Agentes Comunitários de Saúde (ACS) lotados na ESF escolhida, tendo em sua totalidade o número de 10 (dez) ACS. A amostra foi selecionada a partir da assinatura do Termo de Consentimento Livre e Esclarecido (TCLE) no qual, 9 (nove) ACS aceitaram participar do trabalho. O instrumento de coleta de dados utilizado, foi um questionário acerca da tuberculose, elaborado pelos pesquisadores, com base na cartilha do Ministério da Saúde "Tuberculose: informações para agentes comunitários de saúde", ano 2017.

O referido instrumento possuiu 14 perguntas de múltipla escolha, com apenas uma assertiva correta. As questões versavam sobre definição, etiologia, modo de transmissão, quadro clínico, diagnóstico, tratamento, tratamento diretamente observado (TDO), orientações aos contactantes, medidas de prevenção e atribuições dos ACS.Após aprovação pelo Comitê de Ética e Pesquisa em Seres Humanos, sob o no do parecer 2.626.336 e CAAE: 87988718.0.0000.5701, foi executada a capacitação dos agentes comunitários de saúde (ACS) sobre o tema "tuberculose".

A aplicação do instrumento de pesquisa e intervenção ocorreu em três momentos:Inicialmente foi aplicado o questionário pré-teste com o objetivo de quantificar o conhecimento prévio dos ACS;Após o preenchimento, as autoras ministraram uma capacitação sobre tuberculose, com utilização de material didático apoio, onde foram sanadas todas as dúvidas acerca da temática;Por conseguinte, o questionário foi reaplicado e intitulado como pós-teste, com intuito de avaliar os conhecimentos adquiridos após a explanação.

As informações obtidas, foram tabuladas e inseridas no banco de dados do programa Microsoft Excel; posteriormente, através do programa BioEstat 5.0. As variáveis quantitativas foram avaliadas por meio do teste T-Student e expressas sob a forma de média \pm desvio padrão e representadas em gráficos.

As informações obtidas somente foram utilizadas nesta pesquisa, não sendo divulgada qualquer informação que possa levar a identificação do participante. A privacidade dos pesquisados, bem como os 
dados coletados, permanecem mantidos em sigilo, conforme as normas da Resolução 466/12 do CONEP. Nenhum material biológico foi coletado e os questionários foram arquivados com os pesquisadores em local seguro e serão incinerados após 5 anos da finalização da pesquisa.

\section{RESULTADOS}

Foram avaliados o conhecimento sobre tuberculose de nove (09) agentes comunitários de saúde, sendo $88,8 \%$ (8/9) do sexo feminino e $11,1 \%$ (1/9) do sexo masculino, com idades idade variando de 28 a 49 anos, e a prevalência de escolaridade foi o nível superior incompleto $(n=3)$ (Tabela 1).

Tabela 1 - Características demográficas dos Agentes Comunitários de Saúde de uma Unidade Saúde da Família, Belém, 2018.

\begin{tabular}{cll}
\hline Variáveis & N & $\%$ \\
\hline Sexo & 8 & 88,8 \\
\hline Feminino & 1 & 11,1 \\
\hline Masculino & 9 & 100 \\
\hline Total & & \\
\hline Idade & 2 & 22,2 \\
\hline 20 - 30 anos & 3 & 33,3 \\
31 - 40 anos & 4 & 44,4 \\
41 - 50 anos & - & - \\
\hline 51 - 60 anos & 9 & 100 \\
\hline Total & & \\
\hline Escolaridade & - & - \\
\hline Ensino fundamental incompleto & 1 & 11,1 \\
Ensino fundamental completo & 2 & 22,2 \\
Ensino médio incompleto & 2 & 22,2 \\
Ensino médio completo & 3 & 33,3 \\
Ensino superior incompleto & 1 & 11,1 \\
Ensino superior completo & 9 & 100 \\
\hline Total & &
\end{tabular}

Fonte: Oliveira GS, et al., 2020.

A análise do conhecimento prévio dos ACS na aplicação do pré-teste, evidenciou que todos os participantes possuem domínio nos eixos temáticos no que tange à definição, modo de transmissão, quadro clínico e prevenção da tuberculose, representando em 100\% ( $n=9)$ do número de ACS. Nos demais tópicos, identificou-se uma discrepância no conhecimento prévio entre os participantes.

Sobre a atribuição dos ACS na prevenção de TB e as orientações aos contactantes, $88,8 \%$ ( $n=8)$ responderam corretamente o quesito proposto, bem como o tratamento da TB com $77,7 \%(n=7)$ de acerto e sobre o conhecimento do diagnóstico da doença com $66,6 \%(n=6)$ de acerto. Os eixos com maior dificuldade de conhecimento foram relacionados ao tratamento diretamente observado (TDO) em $55,5 \%$ $(n=5)$ e etiologia da TB com 44,4\% ( $n=4)$ (Gráfico 1). 
Gráfico 1 - Conhecimento dos agentes comunitários de saúde sobre tuberculose no Pré-Teste de acordo com os eixos temáticos.

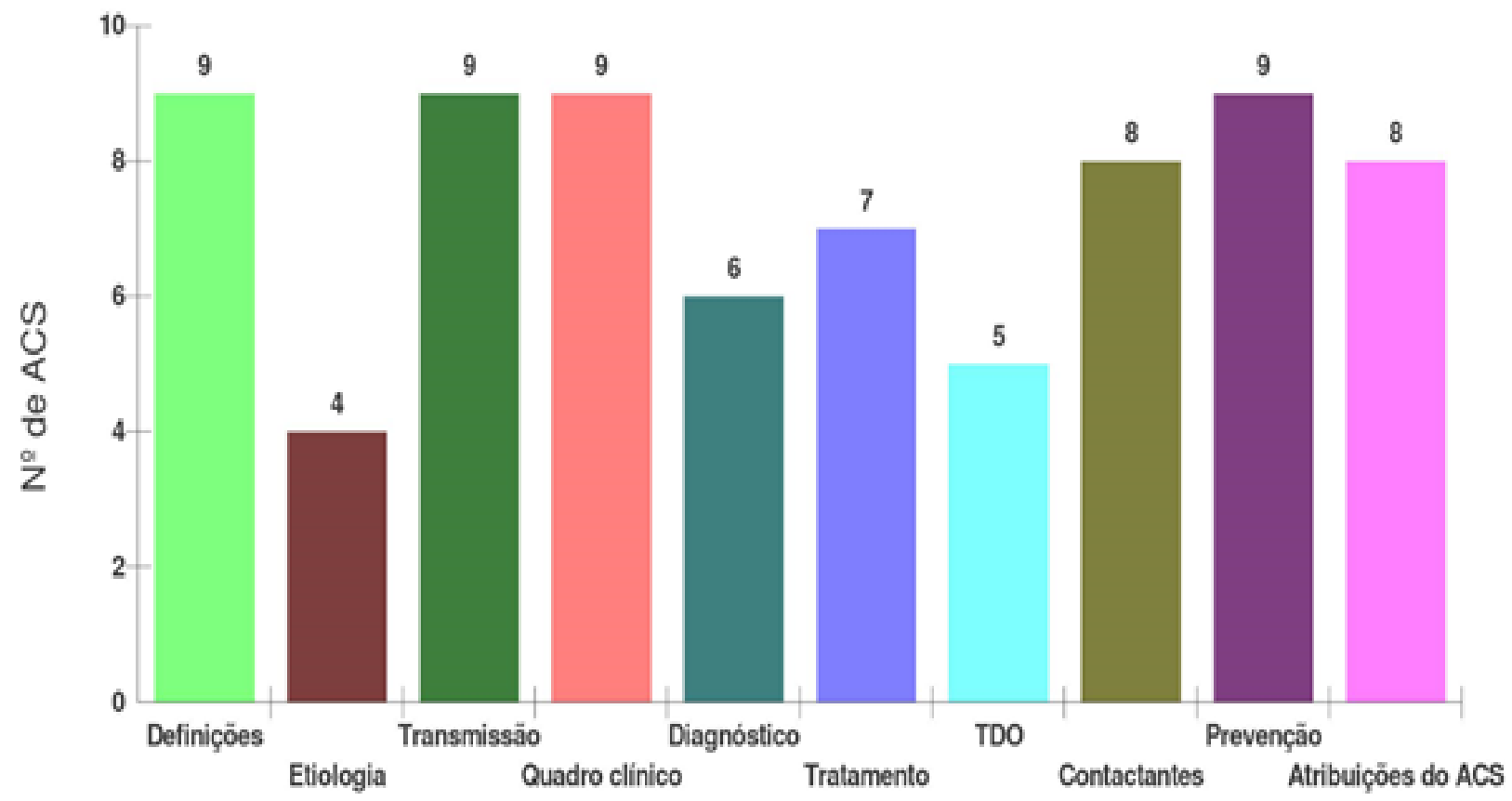

Legenda: ACS: agente comunitário de saúde; TDO: tratamento diretamente observado.

Fonte: Oliveira GS, et al., 2020.

Os resultados obtidos no pós-teste constatam uma equivalência nos conhecimentos adquiridos dos agentes comunitários de saúde após a capacitação sobre tuberculose, de modo que nos quesitos sobre tratamento da TB e orientações aos contactantes houveram acerto de $88,8 \%(n=8)$ do número total de ACS participantes (Gráfico 2).

Gráfico 2 - Conhecimento dos agentes comunitários de saúde sobre tuberculose no pós-teste de acordo com os eixos temáticos.

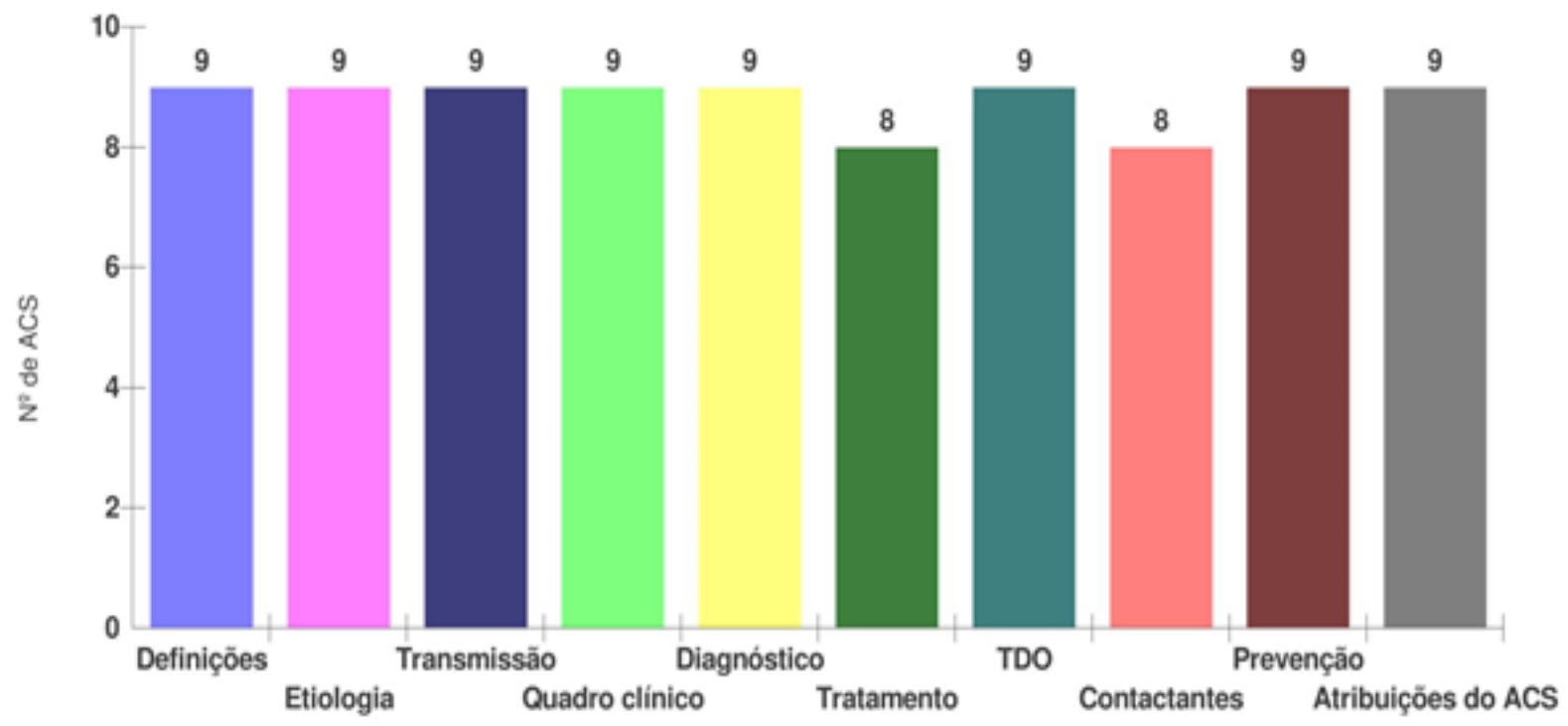

Legenda: ACS: agente comunitário de saúde; TDO: tratamento diretamente observado.

Fonte: Oliveira GS, et al., 2020. 
A comparação do desempenho individual dos ACS na aplicação do pré-teste e pós-teste possui significância estatística ( $p$ valor $=0,027$, Média $\pm 1,77$; $D P \pm 1.98$ ), o que evidenciou que a capacitação sobre tuberculose oferecida aos ACS foi satisfatória. Dessa forma, os agentes comunitários de saúde estão aptos para o reconhecimento de pacientes sintomático-respiratórios suspeitos de tuberculose (Gráfico 3 ).

Gráfico 3 - Comparação de desempenho individual dos agentes comunitários de saúde no pré e pós-teste.

\section{$\triangle$ PRÉ-TESTE}

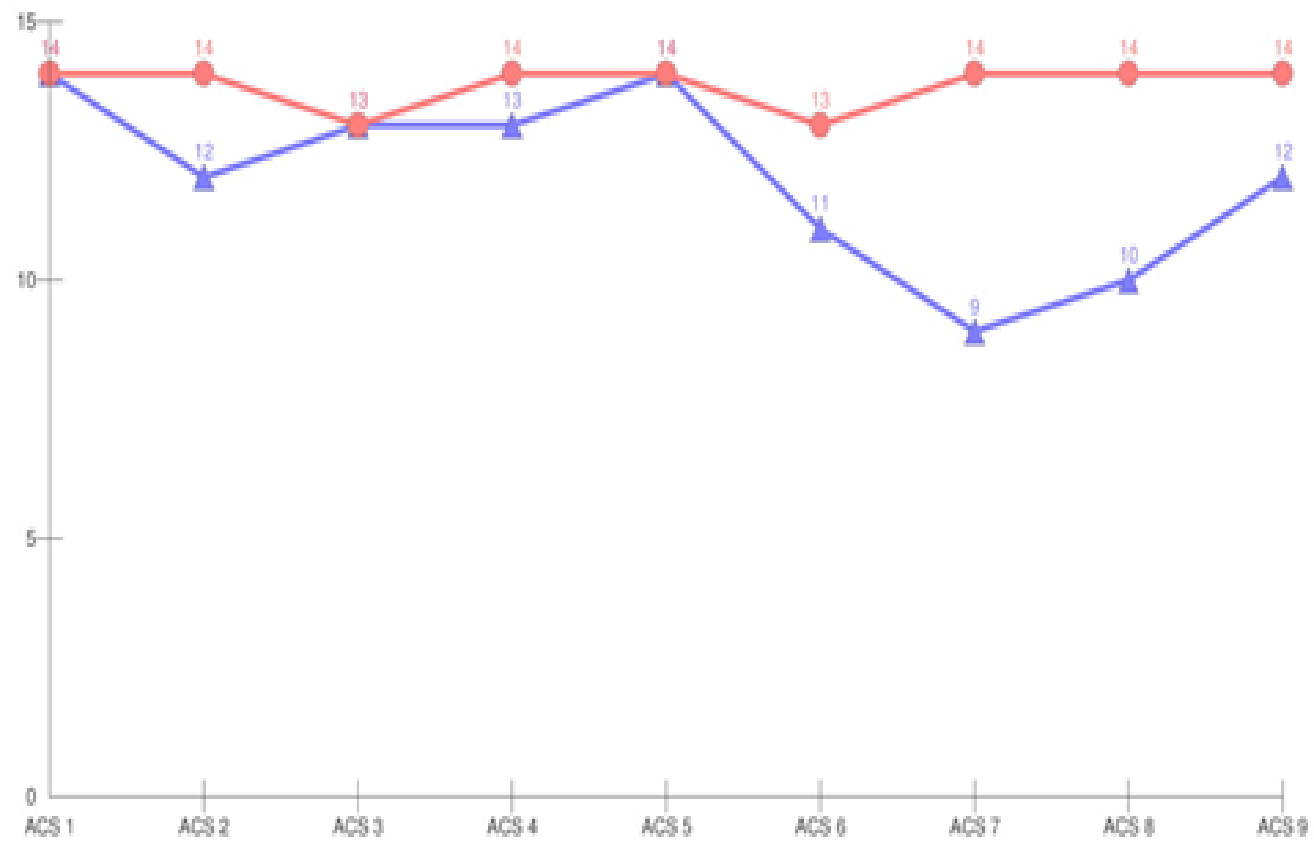

\section{Agentes comunitários de saúde}

Legenda: ACS: agente comunitário de saúde. Fonte: Oliveira GS, et al., 2020.

\section{DISCUSSÃO}

O perfil sociodemográfico dos ACS está em conformidade com outros trabalhos que avaliaram a mesma categoria profissional. A maioria da população deste estudo é do sexo feminino $88,8 \%(n=8)$, fato que é corroborado por demais estudos realizados com a mesma categoria (FAÇANHA MC et al, 2009). Sobre o nível de escolaridade, observou-se na amostra pesquisada, que os ACS possuíam idade entre 20 e 50 anos, sendo que o perfil etário mais prevalente se situou entre 41 e 50 anos, $44,4 \%(n=4)$.

A formação escolar mais prevalente ente os agentes comunitários foi o ensino superior incompleto, correspondendo a 33,3\% ( $n=3)$ do total de entrevistados. Este é um fato de extrema relevância na amostra, pois de acordo com a comparação em nível nacional, é possível observar que os ACS apresentam, majoritariamente, nível de escolaridade superior ao exigido para o cargo segundo a legislação vigente (FAÇANHA MC et al, 2009).

Num estudo realizado, foi evidenciado que a maioria (69\%) dos participantes possuíam o ensino médio completo (SCATOLIN BE et al, 2014). O maior grau de escolaridade parece estar associado a uma maior capacidade do ACS de incorporar novas informações, bem como transmitir o conhecimento para as famílias adscritas à sua microárea.

Ainda que a Organização Mundial de Saúde (OMS) estime que, aproximadamente, um terço da população mundial tenha infecção latente por bactérias pertencentes ao complexo Mycobacterium tuberculosis e que o Brasil esteja entre os países de maior incidência em casos de tuberculose, estudos recentes apontam para uma desaceleração, tanto no número de diagnósticos quanto na mortalidade de pacientes tuberculose (BRASIL, 2018). 
A partir de mudanças ocorridas nos últimos anos, no âmbito da atenção básica, o Agente Comunitário de Saúde passou a ser componente fundamental na promoção de ações de vigilância em saúde e sua atuação no combate e controle da tuberculose tornou-se imprescindível. A capacitação de agentes comunitários contribui para melhorar a busca de sintomáticos respiratórios e consequentemente promover o aumento do número de notificações.

$\mathrm{Na}$ primeira fase deste estudo foi evidenciado que apesar da maioria dos ACS apresentarem conhecimento a respeito da doença, ainda havia a necessidade da implementação de medidas intervencionistas para a ampliação da abrangência de conhecimento dos mesmos.

Essa pesquisa verificou que, no primeiro eixo, 100\% $(n=9)$ dos agentes possuíam conhecimento sobre definição da patologia, modo de transmissão, quadro clínico e prevenção; todos considerados elementos importantes que necessitam ser de conhecimento prévio dos ACS.

Este trabalho encontrou valores superiores aos observados em outra pesquisa, que avaliou o conhecimento de um grupo de agentes comunitários e evidenciou $81 \%$ de acertos de perguntas sobre tuberculose e $84,1 \%$ referente ao eixo de medidas de controle (ROCHA GSS et al, 2015). Tal resultado evidenciou relativa discrepância entre os estudos analisados e os encontrados em outras pesquisas; fato que pode ser relacionado à diferença amostral entre os respectivos estudos, como exemplo, onde o trabalho supracitado apresentou maior quantitativo de participantes.

No segundo eixo da investigação foram evidenciadas respostas parcialmente satisfatórias. No que diz respeito à atribuição do ACS acerca da prevenção da tuberculose junto à comunidade, o acerto do quesito foi de $88,8 \%(n=8)$; sobre as orientações dadas em relação aos contactantes, o número de respostas corretas foi de $77,7 \%(n=7)$, e sobre o conhecimento do diagnóstico da doença foram obtidos $66,6 \%(n=6)$ de acertos.

É possível observar o desconhecimento dos atores do estudo acerca dos métodos diagnósticos, que vão além da baciloscopia de escarro e raio x de tórax. Métodos auxiliares como o histopatológico configuram-se como uma alternativa aos casos difíceis, em que é necessário lançar mão de métodos alternativos para se conseguir fechar o diagnóstico da TB.

Ainda no pré-teste, o eixo em que os ACS demonstraram maior dificuldade de conhecimento está relacionado ao tratamento diretamente observado (TDO) $55,5 \%(n=5)$ e etiologia da TB, com $44,4 \%(n=4)$ de acertos. Constatou-se que no pré-teste, uma parcela dos participantes assinalou a alternativa que se referia a um vírus como o agente etiológico da tuberculose.

Após a capacitação dos atores do estudo, na qual foram fornecidas informações gerais sobre a doença, o número de respostas corretas ao item no pós-teste representou $100 \%(n=9)$ de acerto pelos participantes, comprovando a efetividade da capacitação neste quesito.

No tocante ao TDO, o resultado obtido neste trabalho foi similar ao de outro estudo, que encontrou $59,4 \%$ de acertos neste eixo12. Parte significativa dos ACS acreditavam que o TDO poderia ser realizado por familiares, no entanto, esta prática deve ser realizada exclusivamente por profissionais de saúde, para fins de notificação.

O desconhecimento a respeito do TDO indicou uma falha por parte dos agentes comunitários, que pôde ser sanada com a capacitação e avaliada no pós-teste. Assim, com a correta implementação do TDO, será possível, no futuro, observar melhora do desfecho e diminuição das taxas de abandono do tratamento (ROCHA GSS et al, 2015).

Sobre a identificação de pacientes sintomático-respiratórios (SR), foram verificadas deficiências no préteste, onde foi evidenciada uma taxa de acertos de $66 \%(n=6)$. Neste quesito, o presente estudo contrasta com os dados obtidos por uma pesquisa, que apresentaram escores satisfatórios na identificação dos SR de TB na comunidade e na investigação a partir dos casos índice (SCATOLIN BE et al, 2014). É valido ressaltar que a busca ativa de sintomáticos respiratórios deve ser uma atitude permanente e incorporada à rotina de atividades dos agentes comunitários. 
Sobre os demais quesitos, o pré e pós-teste evidenciaram resultados semelhantes, evidenciando um conhecimento prévio dos sujeitos sobre tais assertivas. Como limitação deste estudo, encontra-se o fato deste ter sido conduzido utilizando-se um questionário não validado para avaliar o conhecimento sobre TB.

\section{CONCLUSÃO}

No presente estudo, a maioria dos ACS da ESF estudada, compareceram e mostraram-se interessados no assunto. Mesmo com relatos verbais de que possuíam muitas dúvidas em relação ao tema, os resultados desta intervenção são considerados satisfatórios e evidenciam que houve melhoria do nível de conhecimento dos ACS, logo, os mesmos encontram-se aptos para o reconhecimento de pacientes sintomático-respiratórios suspeitos de tuberculose.Espera-se que futuramente este estudo sirva de subsídios para implementação de políticas públicas voltadas para a mudança de realidade local, que hajam mais capacitações em diversos temas para os ACS, visando melhora na atuação de seu papel como promotores de saúde e disseminadores de conhecimento junto a população em que atua. Além de, propiciar a realização de novas pesquisas relacionadas a importância da atuação do ACS na saúde pública. Diante disto, considera-se que a abordagem deste estudo seja uma ferramenta para traçar novas estratégias e promover novas capacitações para melhoria da atuação dos ACS.

\section{REFERÊNCIAS}

1. BERALDO AA, et al. Atraso na busca por serviço de saúde para o diagnóstico da Tuberculose em Ribeirão Preto, São Paulo. Ciênc. Saúde coletiva, 2012, 17(11): 10-20.

2. BRASIL. Ministério da Saúde. Cartilha para o Agente Comunitário de Saúde. Tuberculose. Brasília: Ministério da Saúde, 2017.

3. BRASIL. Ministério da Saúde, Secretaria de Vigilância em Saúde. Boletim Epidemiológico. Volume 46. N 9 - 2015.

4. BRASIL. CNES. Cadastro Nacional de Estabelecimentos de Saúde. Disponível em: http://cnes.datasus.gov.br/pages/profissionais/extracao.jsp .

5. FAÇANHA MC, et al. Treinamento da equipe de saúde e busca ativa na comunidade: estratégias para a detecção de casos de TB. J Bras Pneumol. 2009;35(5):449-454.

6. GOLDMAN L, et al. Tratado de Medicina Interna. 22ª́Edição. Rio de Janeiro: ELSEVIER, 2005.

7. LEÃO RNQ, et al. Medicina Tropical e Infectologia na Amazônia. Belém: Samaúma E; 2013.

8. OLIVEIRA GP, et al. Linkage entre SIM e Sinan para melhorar da qualidade dos dados do sistema de informação da tuberculose: a experiência nacional. Cad Saúde Colet. 2010; 18(7); 107-11.

9. ROGERIO WP, et al. Prevalence of infection with Mycobacterium tuberculosis and associated factors in community health workers in Brazil based on the tuberculin skin test. Cad. Saúde Pública 2015, 31(10):35-45.

10. ROCHA GSS, et al. Conocimiento de agentes comunitarios de salud sobre la tuberculosis, medidas de control y tratamiento directamente observado. Cad. Saúde Pública, 2015; 31(7): 20-28.

11. SAMPAIO SN, et al. Perfil epidemiológico da tuberculose no município de Capanema-PA, Norte do Brasil. In: Anais do Congresso brasileiro de medicina de família e comunidade; 2013 mai 29-02; Belém (PA).

12. SCATOLIN BE, et al. Busca de Pacientes Sintomáticos Respiratórios: Atuação do Agente Comunitário de Saúde no controle da tuberculose em município de grande porte, Brasil. Texto Contexto Enferm, Florianópolis, 2014, 23(2): 261-9. 